\title{
Construction of exact solutions and analysis of stability of complex systems by reduction to ordinary differential equations with power nonlinearities
}

\author{
(c) A. A. Kosov ${ }^{1}$, E. I. Semenov ${ }^{2}$
}

\begin{abstract}
Complex systems described by nonlinear partial differential equations of parabolic type or large-scale systems of ordinary differential equations with switching right-side are considered. The reduction method is applied to the corresponding problem for the system of ordinary differential equations without switching. A parametric family of time-periodic and anisotropic on spatial variables exact solutions of the reaction-diffusion system is constructed. The stability conditions of a large-scale system with switching are obtained, which consist in checking the stability of the reduced system without switching. The conditions for the existence of the first integrals for the reduced system of ordinary differential equations expressed by a combination of power and logarithmic functions are found. For the cases of two-dimensional and three-dimensional reduced systems, these conditions are written in the form of polynomial equations relating the system parameters.
\end{abstract}

Key words: complex systems, large-scale switching systems, stability, reaction-diffusion systems, exact solutions, first integrals.

\section{Introduction}

Diffusion processes in multicomponent medium with interacting components are described by systems of nonlinear partial differential equations of parabolic type (PDE PT) [1]. Equations of this kind, called reaction-diffusion systems, are widely used in mathematical biology [2] and in chemical kinetics, in the description of chemical technologies and processes of heat and mass transfer [3], [4], [5]. In the study of parabolic systems of equations, it is important to construct exact solutions [1], [5], since they can be used to describe the operating modes in the modeling of technologies, as well as to verify and configure numerical methods for solving applied problems with boundary conditions. Since non-linear PDE systems are complex objects to study, the reduction method is usually applied to systems of ordinary differential equations (ODE) to construct exact solutions [1]. We consider a reaction-diffusion system modeled by PDE PT with power nonlinearities characterizing the reaction of the mixture components. The use of a special type of ansatz [6] in section 2 allows us to reduce the construction of the PDE PT system solution to a similar problem for two ODE systems, which can be solved sequentially. The first ODE system is nonlinear with right-hand sides represented by combinations of power nonlinearities (ODE PN). The second ODE system is linear with coefficients depending on the solutions of the first ODE system. Therefore, even the stationary solutions of the first ODE PN system are of interest, since in this case the second ODE system has constant coefficients and is integrable, thus we obtain the exact solutions of the original nonlinear PDE PT system. The efficiency of the proposed approach to the construction of exact solutions

\footnotetext{
${ }^{1}$ Alexander A. Kosov, Leading researcher; Matrosov Institute for System Dynamics and Control Theory of Siberian Branch of Russian Academy of Sciences (ISDCT SB RAS); Post Box 292, 134, Lermontov Str., Irkutsk, 664033, Russian Federation, http://orcid.org/0000-0003-1352-1828, email: kosov_idstu@mail.ru

${ }^{2}$ Eduard I. Semenov, Senior researcher; Matrosov Institute for System Dynamics and Control Theory of Siberian Branch of Russian Academy of Sciences (ISDCT SB RAS); Post Box 292, 134, Lermontov Str., Irkutsk, 664033, Russian Federation, http://orcid.org/0000-0002-9768-9945, email: edwseiz@gmail.com
} 
of PDE PT is illustrated by an example of a nonlinear system of 4 equations with 4 spatial coordinates, for which a parametric family of time-periodic and space-anisotropic solutions given by explicit formulas is obtained. In section 3, we consider a large-scale system [7], consisting of several subsystems and the relationships between them, described by ordinary differential equations. Subsystems are described by homogeneous equations with switching of the right parts, interrelations between subsystems can also be switched. Such systems, called switching systems or hybrid systems, have been intensively studied in the last decade [8], [9], [10]. Applying in this section the Matrosov comparison method [11], we reduce the problem of stability of zero solution of a complex system with switching to a similar problem for a much more simple system of ODE PN without switching with the right parts, represented by combinations of power nonlinearities of the same kind that was obtained in section 2. Thus, the properties of the ODE PN system with power nonlinearities, as shown in sections 2 and 3, are transferred to significantly more difficult to study classes of systems modeled by PDE PT and large-scale switching ODE systems. In addition, such ODE PN systems are of independent interest, as they are used in mathematical biology as models of interacting species [2]. Therefore, the study of the ODE PN system and the identification of qualitative properties of its solutions is important for a wider class of differential equations and can be extended to significantly more general and complex systems. The remaining part of the paper is devoted to the study of ODE PN systems with right-hand sides represented by combinations of power nonlinearities. Section 4 deals with the construction of stationary (time-independent) exact solutions and the first integrals of the reduced ODE PN system. Stationary solutions of ODE PN are found from a linear-quadratic system of equations for which a number of cases of nontrivial solvability are considered. Here we obtain conditions on the parameters of the ODE PN system, under which it has explicit first integrals, given by combinations of power and logarithmic functions from phase variables. A number of examples illustrating the results are given.

\section{Reduction to the ODE system and the construction of exact solutions of reaction-diffusion system}

Consider the system of $N$ quasilinear PDE PT

$$
\frac{\partial u_{k}}{\partial t}=\nabla \cdot\left(u_{k}^{\lambda_{k}} \nabla u_{k}\right)+u_{k}^{1-\lambda_{k}} \sum_{j \neq k} \alpha_{k j}(t) u_{j}^{\lambda_{j}},
$$

where $u_{k} \triangleq u_{k}(t, \mathbf{x})$ - are the sought functions; $\mathbf{x} \in \mathbb{R}^{n}-$ is the vector of independent spatial variables, $n \in \mathbb{N}, n \geq 2 ; t \in[0,+\infty)$ - is time; $k=1,2, \ldots, \mathrm{N}, \mathrm{N} \in \mathbb{N} ; \nabla$ - is the gradient operator; $\alpha_{k j}(t)$ - are known functions of time; and the real parameters $\lambda_{k}$ represent nonlinearity of the medium.

We regard the required functions $u_{k}(t, \mathbf{x})$ as the concentrations of interacting components of some mixture of substances, while the known functions $\alpha_{k j}(t)$ characterize the rates of occurring reactions. We cover the situation when functions $\alpha_{k j}(t)$ vanish identically for some $k$ and $j$.

In order to construct exact solutions for system (2.1), we use the ansatz:

$$
u_{k}(t, \mathbf{x})=\psi_{k}(t)\left[W(\mathbf{x})+\varphi_{k}(t)\right]^{1 / \lambda_{k}} .
$$

Here

$$
W(\mathbf{x})=\frac{1}{2}(A \mathbf{x}, \mathbf{x})+(\mathbf{B}, \mathbf{x})+C,
$$


where the nonzero symmetric numerical matrix $A$ of size $n \times n$, the constant vector $\mathbf{B} \in \mathbb{R}^{n}$ and constant $C \in \mathbb{R}$ are to be determined.

Inserting (2.2) into system (2.1) and rearranging, we arrive at

$$
\begin{gathered}
\psi_{k}^{\prime}\left[W(\mathbf{x})+\varphi_{k}\right]+\frac{1}{\lambda_{k}} \psi_{k} \varphi_{k}^{\prime}=\frac{1}{\lambda_{k}} \psi_{k}^{1+\lambda_{k}}\left[W(\mathbf{x})+\varphi_{k}\right] \Delta W(\mathbf{x})+ \\
\quad+\frac{1}{\lambda_{k}^{2}} \psi_{k}^{1+\lambda_{k}}|\nabla W(\mathbf{x})|^{2}+\psi_{k}^{1-\lambda_{k}} \sum_{j \neq k} \alpha_{k j}(t) \psi_{j}^{\lambda_{j}}\left[W(\mathbf{x})+\varphi_{j}\right] .
\end{gathered}
$$

Here $\psi_{k}=\psi_{k}(t), \varphi_{k}=\varphi_{k}(t), \psi_{k}^{\prime}=\frac{d \psi_{k}}{d t}, \varphi_{k}^{\prime}=\frac{d \varphi_{k}}{d t} ; k=1,2, \ldots, \mathrm{N}$. We find from $(2.3)$

$$
|\nabla W(\mathbf{x})|^{2}=\left(A^{2} \mathbf{x}, \mathbf{x}\right)+2(A \mathbf{B}, \mathbf{x})+|\mathbf{B}|^{2}, \quad \Delta W(\mathbf{x})=\operatorname{tr} A-\text { trace of matrix } A .
$$

With these relations and (2.3) the last $N$ equalities rewrite as

$$
\begin{gathered}
\left(\psi_{k}^{\prime}-\frac{\operatorname{tr} A}{\lambda_{k}} \psi_{k}^{1+\lambda_{k}}-\psi_{k}^{1-\lambda_{k}} \sum_{j \neq k} \alpha_{k j}(t) \psi_{j}^{\lambda_{j}}\right)\left(\frac{1}{2}(A \mathbf{x}, \mathbf{x})+(\mathbf{B}, \mathbf{x})+C\right)+ \\
+\psi_{k}^{\prime} \varphi_{k}+\frac{1}{\lambda_{k}} \psi_{k} \varphi_{k}^{\prime}=\frac{\operatorname{tr} A}{\lambda_{k}} \psi_{k}^{1+\lambda_{k}} \varphi_{k}+ \\
+\frac{1}{\lambda_{k}^{2}} \psi_{k}^{1+\lambda_{k}}\left(\left(A^{2} \mathbf{x}, \mathbf{x}\right)+2(A \mathbf{B}, \mathbf{x})+|\mathbf{B}|^{2}\right)+\psi_{k}^{1-\lambda_{k}} \sum_{j \neq k} \alpha_{k j}(t) \psi_{j}^{\lambda_{j}} \varphi_{j}
\end{gathered}
$$

It is straightforward to verify that if the symmetric matrix $A$, the vector $\mathbf{B}$ and the constant $C$ satisfy the next system of algebraic equations:

$$
A=2 \sigma A^{2}, \quad \mathbf{B}=2 \sigma A \mathbf{B}, \quad C=\sigma|\mathbf{B}|^{2},
$$

where $\sigma \neq 0$ is the separation constant, then (2.4) reduces to the system of ordinary differential equations:

$$
\begin{gathered}
\psi_{k}^{\prime}=S_{k} \psi_{k}^{1+\lambda_{k}}+\psi_{k}^{1-\lambda_{k}} \sum_{j \neq k} \alpha_{k j}(t) \psi_{j}^{\lambda_{j}}, \\
\varphi_{k}^{\prime}=\left(\operatorname{tr} A \psi_{k}^{\lambda_{k}}-\lambda_{k} \frac{\psi_{k}^{\prime}}{\psi_{k}}\right) \varphi_{k}+\lambda_{k} \psi_{k}^{-\lambda_{k}} \sum_{j \neq k} \alpha_{k j}(t) \psi_{j}^{\lambda_{j}} \varphi_{j},
\end{gathered}
$$

where

$$
S_{k}=\frac{\operatorname{tr} A}{\lambda_{k}}+\frac{1}{\sigma \lambda_{k}^{2}}
$$

These arguments justify following statement.

T h e o r e m $\mathbf{2 . 1}$ Nonlinear reaction-diffusion system (2.1) admits exact solutions (2.2), where the function $W(\mathbf{x})$ can be an arbitrary polynomial of the form (2.3) with coefficients satisfying (2.5), while the functions $\psi_{k}(t), \varphi_{k}(t)$ are solutions to (2.6), (2.7).

Thus, theorem 2.1 reduce the problem finding of exact solutions for PDE PT (2.1) to solving ODE PN (2.6), (2.7). At the same time, even constant nontrivial solutions of a nonlinear system (2.6) are of interest. The linear autonomous system (2.7) corresponds to such solutions, that can be found in an explicit form. 
E $\mathbf{x} \mathbf{a ~ m ~ p ~ l ~ e ~} \mathbf{2 . 1}$ Consider the system like (2.1) of four equations in the case of four spatial coordinates

$$
\begin{aligned}
\frac{\partial u_{1}}{\partial t} & =\nabla \cdot\left(u_{1}^{1 / 3} \nabla u_{1}\right)+u_{1}^{2 / 3}\left(\frac{3}{2} u_{2}^{1 / 3}-u_{3}^{1 / 3}+\frac{1}{2} u_{4}^{1 / 3}\right) \\
\frac{\partial u_{2}}{\partial t} & =\nabla \cdot\left(u_{2}^{1 / 3} \nabla u_{2}\right)+u_{2}^{2 / 3}\left(\frac{3}{2} u_{1}^{1 / 3}-u_{3}^{1 / 3}+\frac{1}{2} u_{4}^{1 / 3}\right) \\
\frac{\partial u_{3}}{\partial t} & =\nabla \cdot\left(u_{3}^{1 / 3} \nabla u_{3}\right)+u_{3}^{2 / 3}\left(2 u_{1}^{1 / 3}-2 u_{2}^{1 / 3}+u_{4}^{1 / 3}\right) \\
\frac{\partial u_{4}}{\partial t}= & \nabla \cdot\left(u_{4}^{1 / 3} \nabla u_{4}\right)+u_{4}^{2 / 3}\left(\frac{9}{8} u_{1}^{1 / 3}+20 u_{2}^{1 / 3}-\frac{161}{8} u_{3}^{1 / 3}\right) .
\end{aligned}
$$

This system has the following parametric family of exact, periodic in time and anisotropic in spatial variables solutions:

$$
u_{k}(t, \mathbf{x})=\left[W(\mathbf{x})+\varphi_{k}(t)\right]^{3}, \quad k=\overline{1,4}
$$

where

$$
\begin{gathered}
W(\mathbf{x})=-\frac{1}{432} x_{1}^{2}-\frac{1}{108} x_{2}^{2}-\frac{1}{108} x_{3}^{2}-\frac{1}{48} x_{4}^{2}+ \\
+\frac{1}{108} x_{1} x_{2}+\frac{1}{108} x_{1} x_{3}-\frac{1}{54} x_{2} x_{3}+k x_{1}-2 k\left(x_{2}+x_{3}\right)-108 k^{2}, \\
\varphi_{1}(t)=C_{1} \sin t+C_{2} \cos t, \quad \varphi_{2}(t)=C_{1} \sin t+C_{2} \cos t, \\
\varphi_{3}(t)=\left(\frac{358}{193} C_{1}+\frac{48}{193} C_{2}\right) \sin t+\left(\frac{358}{193} C_{2}-\frac{48}{193} C_{1}\right) \cos t \\
\varphi_{4}(t)=\left(\frac{467}{386} C_{1}-\frac{1062}{193} C_{2}\right) \sin t+\left(\frac{467}{386} C_{2}+\frac{1062}{193} C_{1}\right) \cos t .
\end{gathered}
$$

Here $k, C_{1}, C_{2}$ are arbitrary real parameters.

Periodic chemical reactions were discovered by Belousov in the early 50s [12]. Questions of existence and construction of periodic solutions of reaction-diffusion systems are of interest for chemical technology and are studied in a number of papers [13], [14], [15].

\section{Stability analysis of large-scale systems by common vector Lyapunov functions}

Consider a large-scale system with switched subsystems and switched interconnections

$$
\dot{y}_{i}=f_{i}^{\left(s_{i}(t)\right)}\left(y_{i}\right)+\sum_{j \neq i}^{K} f_{i j}^{\left(s_{i j}(t)\right)}(t, \mathbf{y}), \quad i=\overline{1, K} .
$$

Here we denote the state vector for the $i$-th subsystem by $y_{i} \in \mathbb{R}^{n_{i}}$ and $\mathbf{y} \in \mathbb{R}^{n}$ is the full state vector for the large-scale system (3.1). Functions $s_{i}(t), s_{i}:[0,+\infty) \rightarrow M_{i}, M_{i}=\left\{1,2, \ldots, K_{i}\right\}$ determine the switching signal for the $i$-th subsystem. We suppose that functions $f_{i}^{(j)}\left(y_{i}\right)$, $i=\overline{1, K}, j \in M_{i}$ are continuous and homogeneous with order $\varsigma_{i} \geq 1$, where again the rational number $\varsigma_{i}$ has an odd numerator and an odd denominator. Functions $s_{i j}(t), s_{i j}:[0,+\infty) \rightarrow M_{i j}$, $M_{i j}=\left\{1,2, \ldots, K_{i j}\right\}$, determine the switching laws of influence of the $j$-th subsystem on the 
$i$-th one. The functions $s_{i}(t), s_{i j}(t)$ are piecewise-constant, right-sided continuous and have a finite number of point of discontinuities on each finite interval. As far as the functions, which determine the influence of the $j$-th subsystem on the $i$-th one are concerned, we assume that inequalities $\left\|f_{i j}^{(k)}(t, \mathbf{y})\right\| \leq h_{i j}\left\|y_{j}\right\|^{\beta_{j}}$ are satisfied (for all $k \in M_{i j}, t>0, \mathbf{y} \in \mathbb{R}^{n}$ ). Note that the system (3.1) refers to a class of switching systems or hybrid systems, for which urgent problem is to develop effective stability criteria [8], [9], [10]. Suppose that for any $i$-th family of homogeneous differential systems with an order $\varsigma_{i}$

$$
\dot{y}_{i}=f_{i}^{(k)}\left(y_{i}\right), \quad k \in M_{i}
$$

a homogeneous common Lyapunov function (CLF) with an order $\eta_{i}>1$ has been constructed (e.g. on the basis of ([17], Theorem 1)). For all $y_{i} \in \mathbb{R}^{n_{i}}$ the CLF satisfies the following inequalities

$$
\begin{gathered}
a_{1 i}\left\|y_{i}\right\|^{\eta_{i}} \leq V_{i}\left(y_{i}\right) \leq a_{2 i}\left\|y_{i}\right\|^{\eta_{i}}, \quad\left\|\operatorname{grad} V_{i}\left(y_{i}\right)\right\| \leq a_{3 i}\left\|y_{i}\right\|^{\eta_{i}-1}, \\
\left.\dot{V}_{i}\left(y_{i}\right)\right|_{(3.2)} \leq-a_{4 i}\left\|y_{i}\right\|^{\eta_{i}-1+\varsigma_{i}}, \quad a_{k i}>0 .
\end{gathered}
$$

Consider a common vector Lyapunov functions (CVLF), where each component represents a CLF for the corresponding family (3.2):

$$
\mathbf{V}(\mathbf{y})=\operatorname{col}\left(V_{1}\left(y_{1}\right), \ldots, V_{K}\left(y_{K}\right)\right) \text {. }
$$

From (3.3), for the derivative of the $i$-th component of CVLF (3.4) with respect to system (3.1) we obtain the following estimate

$$
\left.\dot{V}_{i}\left(y_{i}\right)\right|_{(3.1)} \leq-a_{4 i} a_{2 i}^{-\frac{\eta_{i}-1+\varsigma_{i}}{\eta_{i}}} V_{i}^{\frac{\eta_{i}-1+\varsigma_{i}}{\eta_{i}}}+V_{i}^{\frac{\eta_{i}-1}{\eta_{i}}} \sum_{j \neq i}^{K} h_{i j} a_{3 i} a_{1 i}^{\frac{1-\eta_{i}}{\eta_{i}}} a_{1 j}^{-\frac{\beta_{j}}{\eta_{j}}} V_{j}^{\frac{\beta_{j}}{\eta_{j}}}, \quad i=\overline{1, K}
$$

Let's use designations:

$$
\begin{gathered}
S_{i}=-a_{4 i} a_{2 i}^{-\frac{\eta_{i}-1+\varsigma_{i}}{\eta_{i}}}, \quad \lambda_{i}=\frac{\eta_{i}-1+\varsigma_{i}}{\eta_{i}}, \quad \mu_{i}=\frac{\eta_{i}-1}{\eta_{i}}, \\
\alpha_{i j}=h_{i j} a_{3 i} a_{1 i}^{\frac{1-\eta_{i}}{\eta_{i}}} a_{1 j}^{-\frac{\beta_{j}}{\eta_{j}}}, \quad \nu_{j}=\frac{\beta_{j}}{\eta_{j}}
\end{gathered}
$$

Using estimates (3.5) and designations (3.6), (3.7) we obtain the comparison system

$$
\dot{u}_{i}=S_{i} u_{i}^{\lambda_{i}}+u_{i}^{\mu_{i}} \sum_{j \neq i}^{K} \alpha_{i j} u_{j}^{\nu_{j}} \equiv \Phi_{i}\left(u_{i}\right), \quad i=\overline{1, K} .
$$

The functions $\Phi_{i}(\mathbf{u})$ are continuous and quasimonotone for all $\mathbf{u} \in \mathbb{R}_{+}^{K}$. Since CVLF (3.4) is positive definite, according to the comparison principle [11] the trivial solution $\mathbf{y}=0$ of (3.1) has the same stability properties as the trivial solution of the comparison system.

T h e o r e m 3.1 If the trivial solution $u=0$ of comparison system (3.8) is stable (asymptotically stable), then the trivial solution $\mathbf{y}=0$ of large-scale system (3.1) is stable (asymptotically stable), at any switching laws $s_{i}(t), s_{i j}(t)$.

Thus, the theorem 3.1 allows to reduce a research of stability of large-scale system with the arbitrary switching laws to the analysis of stability of significantly more simple system (3.8) without switchings. 


\section{Stationary solutions and first integrals}

As shown in section 2, the construction of exact solutions of the reaction-diffusion system leads to the ODE system (2.6), (2.7). We come to more general ODE system (3.8) this same kind in section 3 by applying the vector Lyapunov function method to analyze the stability of a large-scale switching system. Thus, completely different problems led us to study the ODE system (3.8). In addition, this system can meet in other problems and be of independent interest. Therefore, we consider some properties of this ODE system (3.8). In this section of the paper we obtain some exact solutions of the system (3.8) as well as its first integrals.

Stationary solutions of the system (3.8) satisfy the system of algebraic equations: $S_{k} u_{k}^{\lambda_{k}-\mu_{k}}+$ $\sum_{j \neq k} \alpha_{k j} u_{j}^{\nu_{j}}=0$. We will make replacement $Z_{k}=u_{k}^{\nu_{k}}$ and rewrite the system as follows:

$$
S_{k} Z_{k}^{\gamma_{k}}+\sum_{j \neq k} \alpha_{k j} Z_{j}=0
$$

Here for convenience, the symbol $\gamma_{k}=\frac{\left(\lambda_{k}-\mu_{k}\right)}{\nu_{k}}$ is entered. The system (4.1) is nonlinear and in general it is difficult to explore its solvability under arbitrary coefficients, so we consider a number of special cases of interest.

1. Let under all $k=1,2, \ldots, n$ the equality holds $\lambda_{k}=\mu_{k}$. Then (4.1) becomes linear and is written as

$$
Q \mathbf{Z}=-\mathbf{S}
$$

where $\mathbf{Z}=\operatorname{col}\left(Z_{1}, Z_{2}, \ldots, Z_{n}\right)$ is required vector, $\mathbf{S}=\operatorname{col}\left(S_{1}, S_{2}, \ldots, S_{n}\right)-$ is vector of known numbers, and matrix $Q=\left[q_{k j}\right]_{k, j=\overline{1, n}}$ set as follows: $q_{k j}=\alpha_{k j}, k \neq j$ and $q_{k j}=0, k=j$. If $\operatorname{det} Q \neq 0$, then the solution of system (4.2) is issued the only way by Kramer's formulas.

2. Let as $S_{k} \neq 0$ and $\lambda_{k} \neq \mu_{k}$ for all $k=\overline{1, n}$. Then, if matrix of linear part system (4.1) has the form

$$
\Lambda=\left[\begin{array}{cccccc}
0 & \alpha_{12} & 0 & 0 & \ldots & 0 \\
0 & 0 & \alpha_{23} & 0 & \ldots & 0 \\
0 & 0 & 0 & \alpha_{34} & \ldots & 0 \\
\ldots & \ldots & \ldots & \ldots & \ldots & \ldots \\
0 & 0 & 0 & 0 & \ldots & \alpha_{n-1 n} \\
\alpha_{n 1} & 0 & 0 & 0 & \ldots & 0
\end{array}\right]
$$

where $\alpha_{12} \neq 0, \alpha_{23} \neq 0, \ldots, \alpha_{n-1 n} \neq 0, \alpha_{n 1} \neq 0$, the system reduce to only one equation: $\Omega Z_{1}^{\gamma}+Z_{1}=0$, where $\gamma=\prod_{k=1}^{n} \gamma_{k}$ and

$$
\Omega=\frac{S_{n}}{\alpha_{n 1}}\left(-\frac{S_{n-1}}{\alpha_{n-1 n}}\right)^{\gamma_{n}}\left(-\frac{S_{n-2}}{\alpha_{n-2 n-1}}\right)^{\gamma_{n} \gamma_{n-1}} \times \ldots \times\left(-\frac{S_{2}}{\alpha_{23}}\right)^{\prod_{k=3}^{n} \gamma_{k}}\left(-\frac{S_{1}}{\alpha_{12}}\right)^{\prod_{k=2}^{n} \gamma_{k}} .
$$

The latter equation has a nontrivial solution $Z_{1}=\left(-\frac{1}{\Omega}\right)^{\frac{1}{\gamma-1}}$. In this case $Z_{2}, \ldots, Z_{n}$ are defined by formulas

$$
\begin{gathered}
Z_{2}=-\frac{S_{1}}{\alpha_{12}} Z_{1}^{\gamma_{1}}, \quad Z_{3}=-\frac{S_{2}}{\alpha_{23}}\left(-\frac{S_{1}}{\alpha_{12}}\right)^{\gamma_{2}} Z_{1}^{\gamma_{1} \gamma_{2}}, \\
Z_{4}=-\frac{S_{3}}{\alpha_{34}}\left(-\frac{S_{2}}{\alpha_{23}}\right)^{\gamma_{3}}\left(-\frac{S_{1}}{\alpha_{12}}\right)^{\gamma_{3} \gamma_{2}} Z_{1}^{\gamma_{1} \gamma_{2} \gamma_{3}}, \text { etc. }
\end{gathered}
$$

A. A. Kosov, E. I. Semenov. Construction of exact solutions and analysis of stability of complex systems ... 
We proceed to the construction of the first integrals of the ODE system (3.8), in the case when the right part is autonomous $\alpha_{k j}(t)=\alpha_{k j} \equiv$ const. Introduce the notation $\nu_{k}(t)=X_{k}(t)$, $k=\overline{1, n}$ and rewrite the ODE system (3.8) for this case as follows:

$$
\dot{X}_{k}=S_{k} X_{k}^{\lambda_{k}}+X_{k}^{\mu_{k}} \sum_{j \neq k} \alpha_{k j} X_{j}^{\nu_{j}}, \quad X_{k}=X_{k}(t), \quad \dot{X}_{k}=\frac{d X_{k}}{d t} .
$$

We show that the statement is true.

$\mathbf{T} \mathbf{h}$ e o $\mathbf{r} \mathbf{e} \mathbf{m} \mathbf{4} .1$ Let the parameters of a system (4.4) satisfy the following conditions:

1. $\lambda_{k}=1, k=\overline{m+1, n}, m<n$;

2. $\nu_{k}=\mu_{k}-1, k=\overline{m+1, n}$;

3. $\nu_{k}=\mu_{k}-\lambda_{k}, k=\overline{1, m}$

and the following system of algebraic equations:

$$
\sum_{k=1}^{n} A_{k} S_{k}=0, \quad A_{k} \alpha_{k j}+A_{j} \alpha_{j k}=0, \quad k=\overline{1, n}, \quad j=\overline{1, k-1}
$$

has a nontrivial solution $\left(A_{1}, \ldots, A_{n}\right)$. Then system (4.4) has a first integral

$$
J=\sum_{k=1}^{m} \frac{A_{k}}{1-\lambda_{k}} X_{k}^{1-\lambda_{k}}+\sum_{k=m+1}^{n} A_{k} \ln X_{k} .
$$

P r o o f. Derived from the expression (4.6) to the system (4.4) has the form

$$
\begin{aligned}
& \frac{d J}{d t}=\sum_{k=1}^{m} A_{k} X_{k}^{-\lambda_{k}}\left[S_{k} X_{k}^{\lambda_{k}}+X_{k}^{\mu_{k}} \sum_{j \neq k} \alpha_{k j} X_{j}^{\nu_{j}}\right]+ \\
& +\sum_{k=m+1}^{n} A_{k} X_{k}^{-1}\left[S_{k} X_{k}^{\lambda_{k}}+X_{k}^{\mu_{k}} \sum_{j \neq k} \alpha_{k j} X_{j}^{\nu_{j}}\right] .
\end{aligned}
$$

Given the conditions $1-3$, the last equation is rewritten as

$$
\begin{gathered}
\frac{d J}{d t}=\sum_{k=1}^{m} A_{k} S_{k}+\sum_{k=1}^{m} A_{k} X_{k}^{\mu_{k}-\lambda_{k}} \sum_{j \neq k} \alpha_{k j} X_{j}^{\mu_{j}-\lambda_{j}}+ \\
\sum_{k=m+1}^{n} A_{k} S_{k}+\sum_{k=m+1}^{n} A_{k} X_{k}^{\mu_{k}-1} \sum_{j \neq k} \alpha_{k j} X_{j}^{\mu_{j}-\lambda_{j}} \\
=\sum_{k=1}^{n} A_{k} S_{k}+\sum_{k=1}^{m} A_{k} X_{k}^{\mu_{k}-\lambda_{k}} \sum_{j \neq k} \alpha_{k j} X_{j}^{\mu_{j}-\lambda_{j}}+ \\
+\sum_{k=m+1}^{n} A_{k} X_{k}^{\mu_{k}-\lambda_{k}} \sum_{j \neq k} \alpha_{k j} X_{j}^{\mu_{j}-\lambda_{j}}= \\
=\sum_{k=1}^{n} A_{k} S_{k}+\sum_{k=1, n, j=1, k-1}\left(A_{k} \alpha_{k j}+A_{j} \alpha_{j k}\right) X_{k}^{\mu_{k}-\lambda_{k}} X_{j}^{\mu_{j}-\lambda_{j}} .
\end{gathered}
$$

Thus, if the constant $A_{k}, k=\overline{1, n}$ satisfy a system of algebraic equations (4.5), then $\frac{d J}{d t}=0$. That is what we wanted to prove. 
$\mathbf{R}$ e $\mathbf{m}$ a $\mathbf{r} \mathbf{k}$ 4.1 Statement 4.1 remains valid also for cases when

$\lambda_{k} \neq 1$ for all $k=\overline{1, n}$ and $\lambda_{k}=1$ for all $k=\overline{1, n}$. In the first case, the expression for the first integral (4.6) will not contain logarithmic terms, and in the second it will not contain power terms.

$\mathbf{R} \mathbf{e} \mathbf{m}$ a $\mathbf{r} \mathbf{k}$ 4.2 The system (4.5) is a linear homogeneous system $N=\frac{n(n-1)}{2}+1$ equations with respect to $n<N$ unknowns $\left(A_{1}, \ldots, A_{n}\right)$. For nontrivial solutions to exist, it is necessary and sufficient that the rank of the matrix of this system $r$ is less than $n$. For $r=n-1$, the expression (4.6) gives only the first integral whose coefficients $A_{k}, k=\overline{1, n}$ are determined to an arbitrary nonzero factor. For $r<n-1$, the expression (4.6) yields a family of first integrals whose coefficients $A_{k}, k=\overline{1, n}$ depend on $n-r \geq 2$ arbitrary parameters.

E $\mathbf{x} \mathbf{a ~ m ~ p ~ l ~ e ~} 4.1$ Let $n=3$ and the conditions $\lambda_{k} \neq 1$ for all $k=\overline{1,3}$ are satisfied. In this case, the following autonomous ODE system:

$$
\begin{aligned}
& \dot{X}_{1}=S_{1} X_{1}^{\lambda_{1}}+\alpha_{12} X_{1}^{\mu_{1}} X_{2}^{\mu_{2}-\lambda_{2}}+\alpha_{13} X_{1}^{\mu_{1}} X_{3}^{\mu_{3}-\lambda_{3}} \\
& \dot{X}_{2}=S_{2} X_{2}^{\lambda_{2}}+\alpha_{21} X_{1}^{\mu_{1}-\lambda_{1}} X_{2}^{\mu_{2}}+\alpha_{23} X_{2}^{\mu_{2}} X_{3}^{\mu_{3}-\lambda_{3}} \\
& \dot{X}_{3}=S_{3} X_{3}^{\lambda_{3}}+\alpha_{31} X_{1}^{\mu_{1}-\lambda_{1}} X_{3}^{\mu_{3}}+\alpha_{32} X_{2}^{\mu_{2}-\lambda_{2}} X_{3}^{\mu_{3}},
\end{aligned}
$$

according to statement 4.1 has first integral in the form $J=\sum_{k=1}^{3} \frac{A_{k}}{1-\lambda_{k}} X_{k}^{1-\lambda_{k}}$. The unknowns $A_{1}, A_{2}, A_{3}$ satisfy the system of linear algebraic equations

$$
\begin{gathered}
A_{1} S_{1}+A_{2} S_{2}+A_{3} S_{3}=0, \quad A_{1} \alpha_{12}+A_{2} \alpha_{21}=0, \\
A_{1} \alpha_{13}+A_{3} \alpha_{31}=0, \quad A_{2} \alpha_{23}+A_{3} \alpha_{32}=0 .
\end{gathered}
$$

For the existence of nontrivial solutions we require, for example, the equality of all minors of the third order to zero, which leads to the following relations on the parameters of the system:

$$
\alpha_{12} \alpha_{23} \alpha_{31}+\alpha_{13} \alpha_{32} \alpha_{21}=0, \quad \alpha_{23} \alpha_{31} S_{1}+\alpha_{13} \alpha_{32} S_{2}-\alpha_{13} \alpha_{23} S_{3}=0 .
$$

When these equations are hold, the rank of the system matrix (4.8) is 2 and therefore it has a nontrivial solution: $A_{1}=-\frac{\alpha_{31}}{\alpha_{13}} a, A_{2}=-\frac{\alpha_{32}}{\alpha_{23}} a, A_{3}=a$, where $a \neq 0$ is arbitrary constant. Thus, the system (4.7) with parameters satisfying the equality (4.9) has first integral:

$$
J_{1}=\frac{\alpha_{31}}{\left(1-\lambda_{1}\right) \alpha_{13}} X_{1}^{1-\lambda_{1}}+\frac{\alpha_{32}}{\left(1-\lambda_{2}\right) \alpha_{23}} X_{2}^{1-\lambda_{2}}-\frac{1}{1-\lambda_{3}} X_{3}^{1-\lambda_{3}}=\text { const. }
$$

E $\mathbf{x}$ a $\mathbf{m}$ p 1 e 4.2 Consider a system (4.4) with $n=2$, in the form

$$
\dot{X}_{1}=S_{1} X_{1}^{\lambda_{1}}+\alpha_{12} X_{1}^{\mu_{1}} X_{2}^{\nu_{2}}, \quad \dot{X}_{2}=S_{2} X_{2}^{\lambda_{2}}+\alpha_{21} X_{2}^{\mu_{2}} X_{1}^{\nu_{1}} .
$$

We show that there are 4 different variants of conditions on the parameters $S_{i}, \lambda_{i}, \mu_{i}, \nu_{i}$, $(i=1,2), \alpha_{12}, \alpha_{21}$, under which the system (4.10) has the first integrals.

1. Let parameters of system (4.10) satisfy conditions:

$$
\lambda_{1} \neq 1, \quad \lambda_{2} \neq 1, \quad \nu_{1}=\mu_{1}-\lambda_{1}, \quad \nu_{2}=\mu_{2}-\lambda_{2}, \quad \alpha_{21} S_{1}-\alpha_{12} S_{2}=0,
$$

then system (4.10) has first integral

$$
J_{1}=\left(1-\lambda_{2}\right) \alpha_{21} X_{1}^{1-\lambda_{1}}-\left(1-\lambda_{1}\right) \alpha_{12} X_{2}^{1-\lambda_{2}} \equiv \mathcal{C}_{1}
$$


where $\mathcal{C}_{1}$ is arbitrary real constant.

2. Let parameters of system (4.10) satisfy conditions:

$$
\lambda_{1}=\lambda_{2}=1, \quad \nu_{1}=\mu_{1}-1, \quad \nu_{2}=\mu_{2}-1, \quad \alpha_{21} S_{1}-\alpha_{12} S_{2}=0,
$$

then system (4.10) has first integral

$$
J_{2}=\alpha_{21} \ln X_{1}-\alpha_{12} \ln X_{2} \equiv \mathcal{C}_{2},
$$

where $\mathcal{C}_{2}$ is arbitrary real constant.

3. Let parameters of system (4.10) satisfy conditions:

$$
\lambda_{1}=1, \quad \lambda_{2} \neq 1, \quad \nu_{1}=\mu_{1}-1, \quad \nu_{2}=\mu_{2}-\lambda_{2}, \quad \alpha_{21} S_{1}-\alpha_{12} S_{2}=0,
$$

then system (4.10) has first integral

$$
J_{3}=\left(1-\lambda_{2}\right) \alpha_{21} \ln X_{1}-\alpha_{12} X_{2}^{1-\lambda_{2}} \equiv \mathcal{C}_{3},
$$

where $\mathcal{C}_{3}$ is arbitrary real constant.

4. Let parameters of system (4.10) satisfy conditions:

$$
\lambda_{1} \neq 1, \quad \lambda_{2}=1, \quad \nu_{1}=\mu_{1}-\lambda_{1}, \quad \nu_{2}=\mu_{2}-1, \quad \alpha_{21} S_{1}-\alpha_{12} S_{2}=0,
$$

then system (4.10) has first integral

$$
J_{4}=\alpha_{21} X_{1}^{1-\lambda_{1}}-\left(1-\lambda_{1}\right) \alpha_{12} \ln X_{2} \equiv \mathcal{C}_{4},
$$

where $\mathcal{C}_{4}$ is arbitrary real constant.

Acknowledgments. The research was supported by the Russian Foundation for Basic Research (project № 19-08-00746).

\section{REFERENCES}

1. A. D. Polyanin, V.F. Zaitsev, Handbook of Nonlinear Partial Differential Equations. Second Edition, Updated, Revised and Extended, Publisher: Chapman \& Hall/CRC Press, Boca Raton-London-New York, 2012, 1912 p.

2. J. D. Murray, Mathematical biology. I. An Introduction, Springer, 2002, 552 p.

3. J. L. Vazquez, The Porous Medium Equation: Mathematical Theory, Oxford Mathematical Monographs, Oxford: Clarendon Press, 2007.

4. A. D. Polyanin, A. M. Kutepov, A. V. Vyazmin, D. A. Kazenin, Hydrodynamics, Mass and Heat Transfer in Chemical Engineering, Taylor \& Francis, London, New York, 2002, 387 p.

5. V.A. Galactionov, S. R. Svirshchevskii, Subspaces of nonlinear partial differential equations in mechanics and physics, Chapman \& Hall/CRC, 2007, 493 p.

6. G.A. Rudykh, E.I. Semenov, "Construction of exact solutions of the multidimensional quasilinear heat equation", Computational Mathematics and Mathematical Physics, 33:8 (1993), 1087-1097.

7. D. D. Šiljak, Large-scale dynamic systems: stability and structure, North-Holland, New York, 1978, $416 \mathrm{p}$. 
8. Unsolved Problems in Mathematical Systems and Control Theory, Princeton University Press, Princeton, Oxford, 2004.

9. R. Shorten, F. Wirth, O. Mason, K. Wulf, C. King, "Stability criteria for switched and hybrid systems", SIAM Rev, 49:4 (2007), 545-592.

10. Hai Lin, P. J. Antsaklis, "Stability and stabilizability of switched linear systems: a survey of recent results", IEEE Trans. Automat. Contr., 54:2 (2009), 308-322.

11. V. M. Matrosov, Metod vektornyx funkcij lyapunova: analiz dinamicheskix svojstv nelinejnyx sistem [The Method of Vector Lyapunov Functions: Analysis of Dynamical Properties of Nonlinear Systems], Fizmatlit, Moscow, 2001 (In Russ.).

12. B. P. Belousov, Sbornik statej po radiacionnoj medicine za 1958 [The collection of papers on radiation medicine during 1958], Medgiz, Moscow, 1959 (In Russ.), 145 p.

13. J. J. Morgan, S. L. Hollis, "The existence of periodic solutions to reaction-diffusion systems with periodic data", SIAM J. Math. Anal., 26:5 (1995), 1225-1232.

14. Sandro Merino., "Positive periodic solutions for semilinear reaction diffusion systems on $\mathbb{R}^{N "}$, Advances in Differential Equations, 1:4 (1996), 579-609.

15. N. N. Nefedov, E. I. Nikulin, "Existence and Stability of Periodic Solutions for ReactionDiffusion Equations in the Two-Dimensional Case", Modeling and Analysis of Information Systems, 23:3 (2016), 342-348.

16. S. N. Vassilyev, A. A. Kosov, A. I. Malikov, Stability Analysis of Nonlinear Switched Systems via Reduction Method, Proceedings of the 18th IFAC World Congress (Milano, Italy, August 28-September 2 2011), 5718-5723.

17. A. Yu. Aleksandrov, A. A. Kosov, A. V. Platonov, "On the asymptotic stability of switched homogeneous systems", Systems \& Control Letters, 61:1 (2012), 127-133.

Submitted (дату ставит редакиия журнала) 